\title{
Sofrimento mental e desempenho acadêmico em estudantes de Psicologia em Sergipe
}

\author{
Mental suffering and academic performance in Psychology students at Sergipe \\ Sufrimiento mental y desempeño académico en estudiantes de Psicología en Sergipe
}

Recebido: 06/08/2021 | Revisado: 11/08/2021 | Aceito: 13/08/2021 | Publicado: 16/08/2021

\author{
Maria Victória de Araujo Lira \\ ORCID: https://orcid.org/0000-0002-1866-5639 \\ Universidade Tiradentes, Brasil \\ E-mail: victrialira@gmail.com \\ Suelly Cristine de Almeida Santos \\ ORCID: https://orcid.org/0000-0001-5365-8007 \\ Universidade Tiradentes, Brasil \\ E-mail: suellycristinepsicologa@gmail.com \\ Priscilla Campos Vidal \\ ORCID: https://orcid.org/0000-0003-2472-7047 \\ Universidade Tiradentes, Brasil \\ E-mail: priscilla_pcv@hotmail.com \\ Cleberson Franclin Tavares da Costa \\ ORCID: https://orcid.org/0000-0002-0762-1657 \\ Universidade Tiradentes, Brasil \\ E-mail: cleberson_franclin@unit.br \\ Mara Dantas Pereira \\ ORCID: https://orcid.org/0000-0002-5943-540X \\ Universidade Tiradentes, Brasil \\ E-mail: maradantaspereira@gmail.com \\ Míria Dantas Pereira \\ ORCID: https://orcid.org/0000-0002-9774-9717 \\ Universidade Tiradentes, Brasil \\ E-mail: miriadantaspereira@gmail.com \\ Estélio Henrique Martins Dantas \\ ORCID: https://orcid.org/0000-0003-0981-8020 \\ Universidade Tiradentes, Brasil \\ E-mail: estelio@pesquisador.cnpq.br
}

\begin{abstract}
Resumo
O presente trabalho teve como finalidade identificar se há correlação entre desempenho acadêmico e sofrimento mental de estudantes de Psicologia das Instituições de Ensino Superior de Sergipe. A pesquisa foi realizada durante a pandemia da Covid-19, num modelo de estudo transversal e exploratório no período de 08 de março a 22 de abril de 2021, com a aplicação de um formulário de autopreenchimento contendo questões sobre os dados sociodemográficos, autoavaliação de desempenho acadêmico e adaptação do instrumento Self-Report Questionnaire (SRQ-20). O estudo contou com uma amostra de 264 estudantes de Psicologia de 5 Instituições de Ensino Superior de Sergipe. Durante a análise de dados, as associações entre variáveis foram testadas por meio do teste $\mathrm{T}$ de amostras independentes realizadas por meio do IBM SPSS Statistics Base 20.0. A prevalência de indivíduos com indícios de sofrimento psíquico foi de $70,5 \%$, sendo possível concluir diante dos resultados, que podem ser fatores de risco para sofrimento mental: ser do sexo feminino, ter frequência nas aulas igual ou menor a $80 \%$, média ponderada igual ou menor que 8 pontos, reprovação em pelo menos uma disciplina, estar insatisfeito com o curso escolhido, ter pensamento de abandono do curso, por fim, uma autoavaliação negativa acerca do desempenho acadêmico. Esses resultados serão importantes para subsidiar ações voltadas para a prevenção e o cuidado em saúde mental na vida acadêmica de estudantes de psicologia.
\end{abstract}

Palavras-chave: Covid-19; Adoecimento; Estudantes; Psicologia; Graduação; Saúde mental.

\begin{abstract}
The purpose of the work was to identify the correlation between academic performance and mental suffering of Psychology students from higher education institutions in Sergipe. The research was carried out during the Covid-19 pandemic, in an exploratory cross-sectional study model from March 8 to April 22, 2021 with the application of a self-completed form containing questions about sociodemographic data, self-assessment of academic performance and adaptation of the Self-Report Questionnaire (SRQ-20). The study included a sample of 264 Psychology students from 5 higher education institutions in Sergipe. During data analysis, associations between variables were tested using the $\mathrm{T}$ test of independent samples performed using IBM SPSS Statistics Base 20.0. The prevalence of individuals with signs of psychological distress was $70.5 \%$, it was possible to conclude in view of the results, which are risk factors for mental distress: being female, having a frequency equal to or less than $80 \%$, GPA equal or less that 8 points, failing at
\end{abstract}


least one discipline, being dissatisfied with the chosen course, having thought of dropping out of the course, finally, a negative self-assessment about academic performance. These results will be important to support actions aimed at prevention and mental health care in the academic life of psychology students.

Keywords: Covid-19; Illness; Students; Psychology; University graduate; Mental health.

\section{Resumen}

Este estudio tuvo como objetivo identificar si existe una correlación entre el rendimiento académico y el sufrimiento mental de los estudiantes de Psicología de las Instituciones de Educación Superior de Sergipe. La encuesta se realizó durante la pandemia Covid-19, en un modelo de estudio transversal y exploratorio del 8 de marzo al 22 de abril de 2021, con la aplicación de un formulario de autocompletado que contiene preguntas sobre datos sociodemográficos, autoevaluación de desempeño académico y adaptación del instrumento Cuestionario de Autoinforme (SRQ-20). El estudio incluyó una muestra de 264 estudiantes de Psicología de 5 Instituciones de Educación Superior de Sergipe. Durante el análisis de datos, las asociaciones entre variables se probaron mediante la prueba $\mathrm{T}$ de muestras independientes realizada con IBM SPSS Statistics Base 20.0. La prevalencia de individuos con evidencia de angustia psicológica fue del 70,5\%, y de los resultados se puede concluir que pueden ser factores de riesgo de angustia mental: ser mujer, asistir a clases igual o inferior al $80 \%$, promedio ponderado igual o inferior a menos de 8 puntos, reprobar al menos una asignatura, estar insatisfecho con la asignatura elegida, pensar en abandonar la asignatura, finalmente, una autoevaluación negativa sobre el rendimiento académico. Estos resultados serán importantes para apoyar acciones dirigidas a la prevención y atención de la salud mental en la vida académica de los estudiantes de psicología.

Palabras clave: Covid-19; Enfermedad; Estudiantes; Psicología; Graduación; Salud mental.

\section{Introdução}

Ingressar no campo universitário leva o estudante a vivenciar mudanças que se mostram relevantes e profundas à forma como passa a pensar nas mais diversas áreas da sua vida, levando-o ao desenvolvimento intelectual e pessoal (Castro, 2017), porque esta é uma fase complexa e multidimensional, em que necessita de o universitário lidar com questões pessoais e acadêmicas (Sahão, 2019; Almeida, Soares \& Ferreira, 2000).

Entre essas questões, estão a necessidade de se adaptar à rotina de estudo, o distanciamento do convívio familiar e novos contatos sociais, a transição que há da adolescência para a vida adulta, como também para outros, a volta aos estudos após um período longe do ambiente educacional. Outras mudanças que devem ser citadas são a adequação às normas e metodologias de ensino, grupos e pessoas desconhecidas, problemas financeiros, os quais são fatores que podem gerar ansiedade, expectativas, aflições e enfrentamentos (Castro, 2017; Guimarães et al., 2020).

Além de todas as dificuldades já mencionadas, é sabido que a pandemia provocada pela Covid-19 afetou toda a humanidade, alcançando assim também o Ensino Superior no Brasil. Entre as 69 universidades públicas do país, 54 tiveram que fechar suas portas, interrompendo os estudos de 870 mil pessoas (Alves et. al., 2020). A atual pandemia é uma emergência de saúde pública de interesse universal e passou a representar um desafio para a resiliência psicológica (Wang, 2020).

Entre as problemáticas provocadas pela pandemia da Covid-19 à vida estudantil estão o acesso dos estudantes à internet, habilidade dos alunos com o uso do ambiente virtual para estudos, exigindo maior autonomia desses no uso dos recursos digitais para sua aprendizagem (Gusso et. al. 2020). Bem como todas as questões socioemocionais que envolvem docentes, discentes, famílias e sociedade (Silus, 2020).

Anterior aos acontecimentos provocados pela pandemia na vida dos estudantes universitários, Castro (2017), já evidenciava a necessidade de as instituições de ensino dispor de experiências acadêmicas que proporcionassem um amadurecimento pessoal, social e afetivo, como também o profissional e intelectual, porque a universidade se torna uma fonte de influência na vida dos alunos.

Todas essas variáveis se manifestam de forma estressora quando os acadêmicos não conseguem lidar ou realizar as demandas que esse período demanda, apresentando em crenças negativas sobre si, o que acarreta a dificuldade da socialização na academia. As interações realizadas durante a formação atuam como rede de apoio para o estudante; caso isso não ocorra, enfrentar as questões da vida acadêmica podem ser vivenciadas de maneira mais negativa e com mais dificuldades (Ariño \& 
Bardagi, 2018).

Dessa forma, pode-se afirmar que as Instituições de Ensino Superior precisam ofertar serviços que atendam às demandas educativas, psicológicas e sociais dos estudantes (Bisinoto \& Araujo, 2011). Esses autores ainda afirmam que tais serviços necessitam ter como foco ser preventivos, como também de promover, de forma integral, a formação e desenvolvimento do acadêmico.

Diante disso, afirma-se que a vivência acadêmica tem sido um objeto de pouco estudo e discussão, especialmente no curso de Psicologia (Andrade et al., 2016). Visto que, Guimarães et al. (2020), sinalizam que as Diretrizes da legislação dos cursos de graduação de Psicologia no Brasil não abordam as peculiaridades das vivências acadêmicas que esses estudantes vivenciam, já que convivem com o sofrimento psíquico de outros e com assuntos da academia que estão relacionados com a individualidade de cada sujeito.

Os autores já mencionados ainda afirmam que, essas Diretrizes nem consideram a possibilidade de os estudantes serem afetados de alguma forma por estas vivências, ou ainda indicar acompanhamento psicológico para eles. Guimarães et. al. (2020), ainda apontam que o Conselho Federal de Psicologia (CFP, 2017), não dispõe em nenhuma de suas resoluções a utilidade e relevância de se ter acompanhamento psicológico durante a graduação.

À vista disso, é notório que o ambiente acadêmico pode favorecer o adoecimento mental dos estudantes (Castro, 2017). Por isso, é necessário refletir acerca da adaptação dos alunos na universidade, visto que, segundo o autor mencionado, a dificuldade de identificação do aluno para com a universidade influencia diretamente em seu desenvolvimento psicossocial. Assim, é de notória relevância que as Instituições de Ensino Superior reavaliem suas políticas e diretrizes de assistência à saúde mental dos estudantes, visando prevenção e intervenção quando necessário. Destarte, para contribuir nas medidas citadas, as universidades precisam aumentar os investimentos em serviços relacionados à orientação, aconselhamento e acompanhamento dos acadêmicos.

É esperado das Instituições de Ensino Superior proporcionarem um espaço que permita a formulação de pensamento crítico e local de apoio aos universitários, contudo, esse espaço tem se mostrado contraditório onde propicia o adoecimento mental dos discentes (Gomes \& Calixto, 2019). Por essa razão, faz-se necessário abordar os estressores do sofrimento mental dos estudantes.

Os principais sintomas encontrados a partir dos questionários de rastreamento são os níveis de estresse, fadiga e insônia, distúrbios psicossomáticos, irritabilidade, entre outros (Andrade et al., 2016). Ainda de acordo com o mesmo autor, diante da escassez de estudo, ressalta-se a importância do estudo realizado por Gastaud et al. (2006), que teve como resultado a prevalência de 21,1\% de Transtornos Mentais Menores em estudantes de Psicologia.

Os Transtornos Mentais Menores (TMM) se caracterizam como quadros de menor gravidade, porém com maior frequência de transtornos mentais, tendo como principais sintomas a dificuldade de concentração e de tomada de decisões, irritabilidade e fadiga, alterações de memória, além de queixas somáticas como cefaleia e sintomas gastrointestinais (Rocha \& Sassi, 2013).

A partir do estudo realizado por Souza, Caldas e De Antoni (2017), para o reconhecimento dos fatores mais citados quanto ao adoecimento dos estudantes da área da saúde, foi constatado que a "falta de apoio emocional" e a "dificuldade para criar vínculos de amizade" são estressores importantes para o desenvolvimento do sofrimento psíquico. Levando em consideração a essencialidade da rede de apoio no enfrentamento das dificuldades diárias.

Segundo Araújo (2020), observa-se no Ensino Superior o desencadeamento de sintomas que facilitam o sofrimento psíquico, sendo manifestos por meio da melancolia, fobias, depressão, absenteísmo, uso, abuso e dependência de substâncias psicoativas, isolamento social e afetivo, diversos problemas na aprendizagem podendo chegar até a evasão. O sofrimento no ambiente universitário abarca questões relacionadas ao histórico pessoal dos estudantes, diagnósticos médicos, entre outros 
aspectos, como também as esferas coletivas, institucionais e socio estruturais (Leão, Lanni \& Goto, 2019).

As habilidades conformadoras do desenvolvimento pessoal (HCDP), variáveis cognitivas que interferem no comportamento dos universitários, são vitais na organização das atividades acadêmicas, incluindo rotina de estudo e o processo de aprendizagem dos estudantes (Mascarenhas et al., 2020). Dito isso, a adaptação universitária é um processo complexo envolto em diversas situações, como produtividade acadêmica e desenvolvimento institucional, envolvendo um caminho longo de "integração do aluno às vivências acadêmicas, tanto no que diz respeito à aprendizagem técnica quanto ao desenvolvimento pessoal, interpessoal e emocional" (Porto \& Soares, 2017).

A insuficiência de habilidades e competências quanto a organização e enfrentamento das exigências acadêmicas, tais como adaptação a um novo contexto, mudança na rotina de sono, novas necessidades na gestão do tempo de estudo, bem como a pressão acerca do processo de graduação, podem se constituir como estressores do adoecimento estudantil (Ariño \& Bardagi, 2018). O autor ainda afirma que a carência nesse conjunto de habilidades já citado, pode ser vivenciado com maior intensidade pelo aluno.

É necessário considerar, contudo, que o sofrimento psíquico pode não estar inteiramente ligado apenas com a entrada na universidade, mas também com questões da vida pessoal e a maneira como o estudante lida com elas (Chaves et al., 2015). De acordo com o estudo feito por Pinho et al. (2013), a vida acadêmica exige do estudante um posicionamento de maior responsabilidade acerca das tarefas diárias, tornando esse processo de mudança um período que exige mais desses jovens do que estavam anteriormente habituados, podendo desencadear quadros de ansiedade.

Faz-se necessário ressaltar que a presença de tais estressores, por si só, não é determinante de psicopatologias ou de outros sintomas, sejam eles físicos ou emocionais (Souza, Caldas \& De Antoni, 2017). Conforme observa Cunha (p. 33, 2000), "a maioria das manifestações de transtornos psiquiátricos representa variações de diferentes graus de um continuum entre saúde mental e psicopatologia", sendo assim, tais mudanças são evidenciadas pela natureza quantitativa.

Medo, apreensão e preocupação compõem uma combinação complexa de sentimentos que trazem significado ao conceito de ansiedade, podendo se manifestar como uma desordem cerebral ou relacionada a outros problemas clínicos incluindo transtornos psiquiátricos (Costa et al., 2017).

A ansiedade é responsável pela preparação do sujeito para uma ameaça ou perigo iminente juntamente com o medo eles envolvem fatores comportamentais, afetivos, fisiológicos, cognitivos e neurológicos, modulando a percepção do indivíduo acerca do ambiente e gerando respostas específicas (Cardozo et al., 2016).

Apesar de a ansiedade ser caracterizada como uma emoção própria da vivência humana, considerada uma reação de autopreservação, quando em sua condição patológica se apresenta de forma intensa e mais frequente, gerando sintomas que causam grande sofrimento e prejuízo na vida diária, como abandono de emprego, abuso de substâncias e evasão escolar (Leão, 2018).

De acordo com estimativas divulgadas pela Organização Mundial da Saúde (OMS), em fevereiro de 2017, os casos de ansiedade patológica têm aumentado consideravelmente, no qual 9,3\% $(18,657.943)$ da população brasileira foi afetada. Tal transtorno está sendo considerado o mal do último século, ao levar em consideração as mudanças sociais, econômicas e culturais, além das exigências advindas da sociedade moderna, competitiva e tecnológica, que atinge diretamente os sujeitos que estão na transição do fim da adolescência para o início da vida adulta, caracterizando uma etapa marcada por mudanças psicossociais importantes (Cruz et al., 2020).

De acordo com o estudo realizado por Andrade et al. (2016) entre estudantes do curso de Psicologia, os resultados de sua pesquisa revelaram os aspectos concernentes a vivências em geral, organização curricular, relação entre teoria e prática e adaptação à vida universitária como desfavoráveis, segundo a valoração atribuída pelos estudantes a esse tema.

Ainda sobre a mesma pesquisa, que objetivava compreender os fatores mais relevantes quanto ao sofrimento psíquico, 
os autores encontraram como resultado que $90 \%$ (107 respostas) dos estudantes consideram as atividades desenvolvidas durante o curso como desencadeadoras de tal sofrimento. Os autores referenciados questionam a eficácia das Diretrizes Curriculares Nacionais de 2011 para com a singularidade das vivências acadêmicas do estudante de Psicologia, por se encontrar em contato constante com o sofrimento psíquico de outras pessoas.

De acordo com Souza, Caldas e De Antoni (2017), a constante busca por realização, com máxima eficácia e em menor tempo, aliada à falta de estratégias de enfrentamento a eventos adversos, pode vir a ser um dos principais fatores de desgaste físico e emocional. Os mesmos autores anteriormente citados, afirmam ainda que, assim como profissionais, os estudantes universitários estão inclusos numa estrutura organizacional e lhes é requerido o desenvolvimento de habilidades para diversas atividades, a exemplo de relacionar prática e teoria em estágios e avaliações.

É válido ressaltar, ainda, que o estudante traz consigo desde o início da graduação, motivações acerca da profissão que são envoltas em expectativas, experiências e dificuldades. Tais expectativas podem ser frustradas pelas intercorrências no exercício da profissão durante o estágio ou até no estudo de temáticas específicas, o que pode gerar aspectos adoecedores e afetar diretamente a qualidade de vida dos estudantes (Silva et al., 2015).

Diante dessas discussões iniciais, é inegável a relevância do assunto aqui tratado para o meio acadêmico e qualidade de vida do estudante, além de levar em consideração a insuficiência de estudos epidemiológicos, no Brasil, sobre morbidade psiquiátrica em universitários. Associado a isso, diante do corte temporal do presente estudo - pandemia da Covid-19, torna-se ainda mais relevante o objetivo aqui proposto, de identificar se há correlação entre desempenho acadêmico e sofrimento mental de estudantes de Psicologia das Instituições de Ensino Superior de Sergipe.

\section{Metodologia}

Trata-se de uma pesquisa transversal exploratória, com abordagem quantitativa, que descreve uma realidade por meio de dados numéricos, com o objetivo de avaliar comportamentos individuais ou coletivos (Lakatos \& Marconi, 2010). Esse estudo foi aplicado no período de 8 de março a 22 de abril de 2021, para identificar se há correlação entre desempenho acadêmico e sofrimento mental de estudantes de Psicologia das Instituições de Ensino Superior de Sergipe. O estudo ocorreu em 4 etapas simultâneas: Aplicação do termo de consentimento livre e esclarecido, aplicação do questionário dos dados demográficos contendo 9 questões, aplicação do SRQ-20 (Self-Report Questionnaire) - instrumento criado pela OMS em 1994 e validado em diversos países, inclusive no Brasil. A última seção deste questionário foi composta por nove perguntas referentes à autoavaliação do desempenho acadêmico dos participantes. A pesquisa foi aplicada, de forma remota, por meio do Google Forms ${ }^{\circledR}$ e divulgada nas plataformas digitais das universidades do Estado de Sergipe ao curso de Psicologia, bem como por redes sociais.

O presente estudo contou com a participação de 282 estudantes, no entanto, levando em consideração os critérios de inclusão e exclusão do estudo, foram aceitas as respostas dos alunos que referiram estar com a matrícula ativa nos cursos de Psicologia das Instituições de Ensino Superior do Estado de Sergipe desde o primeiro ao décimo período da graduação, e excluídas as respostas dos estudantes de universidades de outros estados bem como de outros cursos de graduação. Sendo assim, foram retiradas da pesquisa 18 respostas, totalizando assim uma amostra com 264 respostas válidas.

O Google Forms ${ }^{\circledR}$ é uma plataforma digital em que foi utilizada para obtenção dos dados de cada participante pela disponibilização do questionário de forma online. Junto a ele, foi aplicado o Termo de Consentimento Livre e Esclarecido (TCLE), documento de maior importância quanto ao aspecto ético de um projeto de pesquisa, sua utilização é o que garante os direitos dos participantes do estudo. Dessa forma, esse termo foi utilizado para explicar aos participantes todo o processo pelo qual eles irão vivenciar caso aceitem responder ao questionário.

O questionário foi dividido em três etapas. A primeira, trata-se de um formulário composto por 9 perguntas acerca de 
dados demográficos e de identificação dos participantes composto por questões abertas e fechadas.

Na segunda etapa, foi utilizada uma versão adaptada do Self-Reporting Questionnaire (SRQ-20), já validado em diversos países, inclusive no Brasil. De acordo com Lacoponi e Mari (1989) esse instrumento foi criado pela Organização Mundial da Saúde 1994, designado para rastrear transtornos psiquiátricos em estabelecimentos de atenção primária à saúde, especialmente em países em desenvolvimento, no entanto, a utilização desse não se limita ao contexto de atenção primária.

Conforme estrutura fatorial do SRQ-20 no Brasil, por Lacoponi e Mari (1989), as respostas foram agrupadas em quatro questões na categoria humor depressivo-ansioso, seis questões em sintomas somáticos, seis questões para decréscimo da energia vital e quatro questões para pensamentos depressivos. As respostas foram do tipo sim/não, em que cada resposta afirmativa totalizava um ponto, para compor o escore final por meio do somatório desses valores. O SRQ-20 indica que há nível de sofrimento mental quando o total de respostas "sim" é maior ou igual a sete.

A terceira e última etapa do questionário foi composta por 9 perguntas acerca da autoavaliação dos estudantes sobre o desempenho acadêmico, com perguntas de sim ou não, questões em escala Likert, e questões com respostas abertas.

Os dados da pesquisa foram guardados no software Microsoft Excel® 2013, onde também foi realizada a verificação de duplicidade de respostas e exclusão de respostas que não atendiam aos critérios de inclusão da pesquisa. Após isso, os dados foram compilados no programa IBM SPSS Statistics Base 20.0, e as análises foram realizadas no mesmo programa. As médias aritméticas e os desvios-padrão foram calculados para todos os dados contínuos da amostra. As perguntas do instrumento utilizado, SRQ-20, foram transformadas em uma variável para ser comparada no teste $\mathrm{T}$ de amostra independente, cujo objetivo é verificar a significância das correlações.

Assim como toda pesquisa de campo realizada em território brasileiro, a primeira etapa foi a submissão deste estudo à Plataforma Brasil, onde foram analisados os aspectos éticos da pesquisa, com aprovação do Comitê de Ética da Universidade do Estado do Pará (CAAE: 39463920.0.0000.5174). Todos os participantes preencheram um Termo de Consentimento Livre e Esclarecido.

\section{Resultados e Discussão}

264 indivíduos participaram do estudo, sendo $81,4 \%$ do gênero feminino $(\mathrm{n}=215)$ e 18,6\% do gênero masculino $(n=49)$, e todos os alunos são do curso de Psicologia. O percentual de participantes com idades entre 18 e 24 anos foi de 69,3\% e com idades entre 25 e 64 anos foi de 30,7\%, sendo a média da idade 28,2 (DP=10,85). Em relação à ocupação, 176 alunos $(66,7 \%)$ responderam que apenas estudam, enquanto 88 (33,3\%) afirmaram que estudam e trabalham. Quanto ao estado civil, 231 participantes $(87,5 \%)$ responderam ser casados e 33 (12,5\%) estão solteiros. 133 indivíduos (50,4\%) apresentam renda familiar de até 3 salários mínimos, ao passo que 131 (49,6\%) revelam renda familiar de 4 salários mínimos em diante. Apenas 8 participantes eram de outros Estados, portanto 256 (97\%) são de Sergipe.

Em relação à Instituição de ensino, 205 alunos (77,7\%) afirmam estudar na Instituição A e 59 (22,3\%) em outras Instituições de Ensino Superior. 168 participantes $(63,6 \%)$ estão entre o $6^{\circ}$ e $10^{\circ}$ período da graduação, ou seja, na última metade do curso, e 96 alunos $(36,4 \%)$ estão entre o $1^{\circ}$ e $5^{\circ}$ período.

$\mathrm{O}$ SRQ-20 tem como ponto de corte a pontuação $\geq 7$, tendo pontuação mínima de 0 que corresponde à ausência de indicativos de sofrimento mental, e 20 que corresponde a altos indicativos de sofrimento mental (OMS, 1994). A pontuação da amostra estudada apresentou uma variação com mínima de zero e máxima de dezenove (média = 9,44; desvio padrão = 4,690), com intervalo de confiança de (IC 95\%: 8,87 a 10,01). A prevalência de sofrimento mental (pontuação no SRQ-20 $\geq 7$ ) foi de $70,5 \%$ da amostra.

Diante do teste-t independente, pode-se constatar que as participantes do sexo feminino (média $=9,85 ; \mathrm{DP}=4,577$ ) apresentam maiores indicativos de sofrimento mental em comparação aos participantes do sexo masculino (média = 7,67; DP = 
$4,815)$ e ( $(262)=-2,970 ; p<0,05)$. Houve associação positiva entre os indicativos de sofrimento mental e a falta de atividade física (média $=10,26 ; \mathrm{DP}=4,825)$ e $(\mathrm{t}(262)=-2,966 ; \mathrm{p}<0,05)$, aqueles que afirmam realizar atividade física apresentaram média $=8,57 ; \mathrm{DP}=4,393$ e p $<0,05$.

Com relação ao desempenho acadêmico dos estudantes, existe associação significativa entre a assiduidade às aulas ( $<<0,05)$ e o questionário de saúde mental, havendo uma média maior entre aqueles que apresentam frequência de até $80 \%$ (média $=12,15 ; \mathrm{DP}=4,310)$ quando comparado aos alunos com frequência de $81 \%$ a $100 \%$ (média $=8,95 ; \mathrm{DP}=4,595)$ e $(\mathrm{t}(262)=4,137 ; \mathrm{p}<0,05)$. Da mesma forma, os estudantes que revelam média geral ponderada entre 6 e 8 pontos (média $=$ 10,33; DP $=4,683$ ) apresentam maiores índices de indicativos de sofrimento mental, em comparação aos alunos com média geral entre 8,1 e 10 pontos (média $=8,94 ; \mathrm{DP}=4,654 ; \mathrm{p}<0,05$ ). Os estudantes que afirmaram ter reprovado em alguma disciplina na graduação (média $=10,77 ; \mathrm{DP}=4,338 ; \mathrm{p}<0,05$ ) apresentaram associação positiva com o questionário SRQ-20.

Ainda sobre o desempenho acadêmico, os alunos que declararam insatisfação com o curso escolhido (média = 13,0; $\mathrm{DP}=4,383 ; \mathrm{p}<0,05)$ apresentaram uma média maior de indícios de sofrimento mental quando comparado com os que se consideram satisfeitos com o curso (média $=8,92 ; \mathrm{DP}=4,510 ; \mathrm{p}<0,05$ ). Quanto ao pensamento de abandono do curso, os estudantes que responderam "sim" (média $=13,78 ; \mathrm{DP}=3,641 ; \mathrm{p}<0,05$ ) indicam maior sofrimento mental que os participantes que responderam "não" (média $=8,95 ; \mathrm{DP}=4,545 ; \mathrm{p}<0,05$ ). Ao questionarmos sobre como o aluno avalia seu desempenho na graduação, os que responderam como desempenho "ruim" (média $=14,27$; DP $=2,898 ; \mathrm{p}<0,05$ ) revelam maior indicativo de sofrimento, em paralelo aos alunos declaram como "bom" (média $=9,0 ;$ DP $=4,577$ ). Os demais dados referentes ao desempenho acadêmico constam na Tabela 1.

A partir do mesmo teste amostral mostrou que, em média, os alunos com idade entre 18 e 24 anos (média = 9,97; DP= 4,739) quando comparados aos alunos com idades de 25 a 64 anos (média $=8,26$; DP= 4,378) não apresentam relevância para o questionário de avaliação de saúde mental, levando em consideração que t (262) =2,763 e p>0,05.

Em relação à influência da Instituição de Ensino Superior nos resultados do SRQ-20, não houve correlação positiva $(\mathrm{t}(262)=0,225 ; \mathrm{p}>0,05)$ na comparação entre os grupos, "Instituição A" e "Outras Instituições", apesar de os dois grupos terem apresentado indicativos de sofrimento mental. As variáveis sociodemográficas, ocupação, período da graduação e renda não apresentaram associação.

Tabela 1 - Tabela descritiva dos dados referentes ao desempenho acadêmico.

\begin{tabular}{lll}
\hline & Número & \% \\
\hline Frequência em aulas & & \\
Até 80\% & 41 & 15,5 \\
De 81\% a 100\% & 223 & 84,4 \\
Média geral ponderada & - & - \\
6 a 8 pontos & 69 & 26,1 \\
8,1 a 10 pontos & 179 & 67,8 \\
Reprovação em alguma disciplina & - & - \\
Sim & 73 & 27,6 \\
Não & 191 & 72,3 \\
Satisfação com o curso escolhido & - & - \\
Sim & 34 & 12,8 \\
Não & 230 & 87,1 \\
Pensamento de abandono do curso & - & - \\
Sim & 27 & 10,2 \\
Não & 237 & 89,7 \\
\hline
\end{tabular}

Fonte: Autores, com base na pesquisa aplicada. 
Tabela 2 - Estatísticas descritivas do somatório de pontuação SRQ-20.

\begin{tabular}{lccccc}
\hline \multicolumn{1}{c}{ Variáveis } & n & Mínimo & Máximo & Média & $\begin{array}{c}\text { Desvio } \\
\text { padrão }\end{array}$ \\
\hline $\begin{array}{l}\text { Escore } S R Q-20 \\
\text { Somatório de pontuação }\end{array}$ & - & - & - & - & - \\
\hline
\end{tabular}

Fonte: Autores, com base na pesquisa aplicada.

Tabela 3 - Estatísticas descritivas da pontuação individual ao SRQ-20.

\begin{tabular}{ccccc}
\hline Respostas & Frequência & Percentual & $\begin{array}{c}\text { Porcentagem } \\
\text { Válida }\end{array}$ & $\begin{array}{c}\text { Porcentagem } \\
\text { Acumulada }\end{array}$ \\
\hline 0 & 5 & 1,9 & 1,9 & 1,9 \\
1 & 9 & 3,4 & 3,4 & 5,3 \\
2 & 10 & 3,8 & 3,8 & 9,1 \\
3 & 6 & 2,3 & 2,3 & 11,4 \\
4 & 21 & 8,0 & 8,0 & 19,3 \\
5 & 8 & 3,0 & 3,0 & 22,3 \\
6 & 19 & 7,2 & 7,2 & 29,5 \\
7 & 16 & 6,1 & 6,1 & 35,6 \\
8 & 11 & 4,2 & 4,2 & 39,8 \\
9 & 19 & 7,2 & 7,2 & 47,0 \\
10 & 26 & 9,8 & 9,8 & 56,8 \\
11 & 18 & 6,8 & 6,8 & 63,6 \\
12 & 25 & 9,5 & 9,5 & 73,1 \\
13 & 14 & 5,3 & 5,3 & 78,4 \\
14 & 14 & 5,3 & 5,3 & 83,7 \\
15 & 15 & 5,7 & 5,7 & 89,4 \\
16 & 9 & 3,4 & 3,4 & 92,8 \\
17 & 12 & 4,5 & 4,5 & 97,3 \\
18 & 5 & 1,9 & 1,9 & 99,2 \\
19 & 2 & 0,8 & 0,8 & 100,0 \\
Total & 264 & 100,0 & 100,0 & - \\
\hline
\end{tabular}

Fonte: Autores, com base na pesquisa aplicada.

Este estudo objetivou identificar se há correlação entre desempenho acadêmico e sofrimento mental de estudantes de Psicologia das Instituições de Ensino Superior de Sergipe. Para tanto, obteve-se uma amostragem composta unicamente por estudantes de Psicologia, levando em consideração os critérios de inclusão na pesquisa embasado no argumento trazido por Andrade et al. (2016), o qual afirma a precariedade de estudos na literatura que investiguem aspectos das vivências acadêmicas no curso de Psicologia.

De acordo com Lhullier, Roslindo e Moreira (2013) a psicologia no Brasil se apresentava majoritariamente composta por mulheres, sendo que nove entre dez pessoas que exerciam a profissão no Brasil eram mulheres, pelo menos desde o final da década de 1980. No Estado de Sergipe, de acordo com dados disponibilizados pelo site do Conselho Federal de Psicologia, a profissão segue majoritariamente feminina sendo 2.625 psicólogas cadastradas no conselho regional e 527 psicólogos. Esses dados corroboram a análise de características da amostra havendo uma maioria de participantes do sexo feminino (81,4\%).

O estudo também indicou maiores níveis de sofrimento mental para o sexo feminino, dado semelhante ao de estudos anteriores realizados com graduandos da área da saúde (Silva \& Costa, 2012; Teixeira et. al. 2020). A literatura associa isso aos múltiplos papéis exercidos pela mulher na sociedade, acompanhado de alterações hormonais, que interferem no estado de humor. É encontrado na literatura referenciada em diversos estudos que as mulheres são mais predispostas que homens a desenvolver sofrimento mental (Lima \& Brito, 2018; Miranda, Tarasconi \& Scortegagna, 2008; Rocha et. al. 2010; Pinho \& Araújo, 2012).

Além disso, essa pesquisa apontou maior prevalência de sofrimento mental em estudantes com média inferior ou igual 
a 8 pontos e frequência menor ou igual a $80 \%$, já no estudo de Brandão (2016) não houve correlação significativa entre média de nota dos alunos e assiduidade às aulas com o nível de estresse. A pesquisa feita por Santos et. al. (2013) com estudantes de Psicologia e Odontologia de uma universidade particular, aponta que os alunos apresentaram níveis de satisfação com o curso superior à média, dado esse que corrobora com a pesquisa em questão. No entanto, apesar do alto número de satisfação ( $\mathrm{n}=230$; média=87,1\%), aqueles que denotam insatisfação com o curso apresentaram maiores indicativos de sofrimento mental.

Em nossa amostra, houve uma maior prevalência de alunos que consideram seu desempenho acadêmico como "bom", contudo, aqueles que consideram seu desempenho como "ruim" indicam maior sofrimento mental, sendo essa variável a maior média no estudo realizado. Em congruência com o atual dado, uma pesquisa feita por Brandão (2016) os indivíduos que consideram seu desempenho acadêmico insuficiente (66,7\%) apresentam maior prevalência de transtornos mentais comuns (TMC), assim como 50\% da amostra também apresenta pensamento de abandono do curso associado à TMC.

Concernente a essa variável, os estudantes que apresentam maiores indícios de sofrimento mental são aqueles que revelam predisposição à evasão. Na análise citada por Casanova et al. (2018), as variáveis pessoais e acadêmicas impactam diretamente na intenção de abandono do curso. Analogamente à literatura referenciada por Nogueira (2017), os problemas de saúde mental interferem no bem-estar dos estudantes de Ensino Superior, afetando o percurso acadêmico e aumentando a taxa de abandono.

É possível apontar uma relação direta entre reprovação em alguma disciplina e níveis de sofrimento psíquico. Ao correlacionar com a pesquisa realizada por Cristo et al. (2019), em que os autores buscaram compreender a percepção de estagiários na área da saúde quanto às prováveis causas do sofrimento mental e suas consequências, concluiu que reprovação e desnivelamento são efeitos/consequências dos problemas de saúde mental.

Outro dado importante foi que 70,5\% da amostra deste estudo apresentou níveis significativos de sofrimento mental. Outros estudos que utilizaram o mesmo instrumento para avaliar o sofrimento psíquico entre graduandos apresentaram os seguintes resultados: entre alunos da área da saúde, incluindo Psicologia, 20\% da amostra apresentou pré-disposição à transtornos mentais comuns (Silva \& Costa, 2012); com estudantes de graduação em geral 39,97\% apresentaram sofrimento psicológico significativo (Padovani et al., 2014). Já em estudo mais recente, realizado apenas com estudantes de Medicina, a prevalência foi de $62,8 \%$ (Teixeira et al., 2020).

\section{Considerações Finais}

A relação entre sofrimento mental e desempenho acadêmico apresentou-se significativa na amostra estudada. Ademais, foi possível concluir diante dos resultados, que são fatores de risco: ser do sexo feminino, ter frequência igual ou menor a $80 \%$, média geral ponderada igual ou menor que 8 pontos, reprovação em pelo menos uma disciplina, estar insatisfeito com o curso escolhido, ter pensamento de abandono do curso, por fim, uma autoavaliação negativa acerca do desempenho acadêmico. Apesar disso, todos os grupos de variáveis da amostra apresentam algum nível de sofrimento mental.

Esse estudo pode sinalizar uma relação entre a pandemia da COVID-19 e os altos níveis de sofrimento mental em estudantes de Psicologia do Estado de Sergipe. Isso se dá pelo fato de a pesquisa ter sido realizada durante esse período, no entanto, esse estudo traz como limitação a ausência de dados, da mesma amostra, anteriores ao período pandêmico. Ainda enquanto limitação seria necessário o uso de outros instrumentos validados para avaliação do desempenho acadêmico e de sofrimento mental, a fim de tornar os dados mais precisos.

Diante dos resultados obtidos, é de grande importância que sejam realizadas pesquisas para avaliar qualitativamente a relação entre adoecimento mental e a experiência da vida acadêmica, com estudantes de Psicologia, tendo em vista a escassez de estudos com esse público específico. A partir dos resultados desta pesquisa, é possível fornecer contribuições para o desenvolvimento de melhorias no âmbito de apoio psicossocial aos alunos de Psicologia em Sergipe, como ações preventivas 
para indícios de sofrimento mental.

\section{Referências}

Almeida, L. S., Soares, A. P. C. \& Ferreira, J. A. G. (2000). Transição e adaptação à Universidade: Apresentação de um Questionário de Vivências Acadêmicas (QVA). Psicologia, 14(2): 189-208.

Andrade, A. S., Tiraboschi, G. A., Antunes, N. A., Viana, P. V. B. A., Zanoto, P. A. \& Curilla, R. T. (2016). Vivências Acadêmicas e Sofrimento Psíquico de Estudantes de Psicologia. Psicologia, Ciência e Profissão, 36(4): 831-846.

Antoniazzi, A. S., Dell'Aglio, D. D. \& Bandeira, D. R. (1998). O conceito de coping: uma revisão teórica. Estudos de Psicologia (Natal), 3(2): $273-294$.

Araújo, C. L. (2020). A constituição da subjetividade de estudantes em práticas de promoção de saúde no contexto da educação superior. Dissertação (Mestrado em educação) Universidade Federal de Alfenas. Minas Gerais.

Arino, D. O. \& Bardagi, M. P. (2018). Relação entre Fatores Acadêmicos e a Saúde Mental de Estudantes Universitários. Psicologia em Pesquisa, 12(3): 4452.

Barros, M. S. P. \& Wilhelm, F. A. (2020). Estratégias de enfrentamento do estresse utilizadas por jovens universitários no extremo norte do país. Revista Brasileira de Iniciação Científica (RBIC), 7(1): 100-117.

Bastos, E. M., Maia, A. M., Oliveira, C. L. F. \& Ferreira, S. N. (2019). Sofrimento Psíquico de Universitários: uma revisão integrativa. Anais do XIV Encontro de Iniciação Científica da UNI7, 8(1).

Bayram, N. \& Bilgel, N. (2008). The prevalence and socio-demographic correlations of depression, anxiety and stress among a group of university students. Social psychiatry and psychiatric epidemiology, 43: 667-672.

Bisinoto, C. \& Marinho-Araujo, C. (2011). Maria. Psicologia escolar na educação superior: atuação no distrito federal. Psicologia em estudo, 16(1): 111-122.

Borba, C. S., Hayasida, N. M. A. \& Lopes, F. M. (2019). Ansiedade social e habilidades sociais em universitários. Psicologia e Pesquisa, 13(3): 119-137.

Brandão, A. S. (2016). Desempenho acadêmico de universitários, variáveis preditoras: habilidades sociais, saúde mental, características sociodemográficas e escolares. Tese (Pós-graduação em Psicologia) Faculdade de Filosofia, Ciências e Letras, Universidade de São Paulo, 208 p.

Cardozo, M. Q., Gomes, K. M., Fan, L. G. \& Soratto, M. T. (2016). Fatores associados à ocorrência de ansiedade dos acadêmicos de biomedicina. Revista Saúde e Pesquisa, 9(2): 251-262.

Casanova, J. R., Fernandez-Castañon, A. C., Pérez, J. C. N., G, A. B. B. \& Almeida, L. S. (2018). Abandono no Ensino Superior: Impacto da autoeficácia na intenção de abandono. Revista Brasileira de Orientação Profissional, 19(1): 41-49.

Castro, V. R. (2017). Reflexões sobre a saúde mental do estudante universitário: Estudo empírico com estudantes de uma instituição pública de Ensino Superior. Revista Gestão em Foco, 9: 380-399.

Cerchiari, E. A. N. (2004). Saúde Mental e Qualidade de vida em estudantes universitários. Campinas, SP [s.n]. Tese (Doutorado em Ciências Médicas) Universidade Estadual de Campinas. Faculdade de Ciências Médicas.

Chaves, E. C. L., Lunes, D. H., Moura, C. C., Carvalho, L. C., Silva, A. M. \& Carvalho, E. C. (2015). Ansiedade e espiritualidade em estudantes universitários: um estudo transversal. Revista Brasileira de Enfermagem, 68(3):504-509.

Conselho Federal De Psicologia. (2013). Uma profissão de muitas e diferentes mulheres: resultado preliminar da pesquisa 2012. 1-30 p.

Costa, D. S., Medeiros, N. S. B., Cordeiro, R. A., Frutuoso, E. S., Lopes, J. M. \& Moreira, S. N. T. (2020). Sintomas de Depressão, Ansiedade e Estresse em Estudantes de Medicina e Estratégias Institucionais de Enfrentamento. Revista Brasileira de Educação Médica, 44(1).

Costa, K. M. V., Sousa, K. R. S., Formiga, P. A., Silva, W. S. \& Bezerra, E. B. N. (2017). Ansiedade em universitários na área da saúde. II CONBRACIS - II Congresso Brasileiro de Ciências da Saúde. Faculdade Maurício de Nassau.

Cristo, F., Farias, I. M. S. U., Cavalcante, A. C., Medeiros, A. L. G., Lima, G. D. O. \& Diogo, W. F. Q. (2019). O Ensino Superior e suas exigências: consequências na saúde mental dos graduandos. Revista Trabalho EnCena. Tocantis, 4(2): 485-505.

Cruz, M. C. N. L. et. al. (2020). Ansiedade em universitários iniciantes de cursos da área da saúde. Brazilian Journal of Health Review, Curitiba, 3(5): 1464414662 .

Cunha, J. A. et. al. (2000). Psicodiagnóstico - V: (5a ed.), Artmed, 33 p.

Division of mental health world health organization geneva. (1994). A User's Guide to the Self Reporting Questionnaire (SRQ).

Faro, A. (2013). Estresse e Estressores na Pós-Graduação: Estudo com Mestrandos e Doutorandos no Brasil. Revista Psicologia: Teoria e Prática, Sergipe, 29(1): 51-60.

Fonaprace. (2016). IV Pesquisa do Perfil Socioeconômico e Cultural dos Estudantes de Graduação: Das Instituições Federais de Ensino Superior Brasileiras, 2014.

Gastaud, M. B. et al. (2006). Bem-estar espiritual e transtornos psiquiátricos menores em estudantes de psicologia: estudo transversal. Revista de Psiquiatria, Rio Grande do Sul, 28(1):12-18. 
Gomes, L. \& Calixto, M. F. (2019). Saúde mental e Ensino Superior: a relação da universidade com o surgimento elou agravo de sofrimento mental em estudantes universitários. Trabalho de Conclusão de Curso (Bacharelado em Enfermagem)- Curso de Enfermagem, Faculdade CESMAC do Sertão, Palmeira dos Índios-Al.

Guimarães, C. C., Moraes, H. A. B., Barboza, M. E. S. \& Mesquita, R. R. (2020). Saúde mental do estudante de Psicologia: Possíveis implicações para sua atuação profissional. Revista Trabalho EnCena, Tocantins, 5(1): 269-284.

Gusso, H. L. et. al. (2020). Ensino Superior em tempos de pandemia: diretrizes à gestão universitária. Educação \& Sociedade, Campinas, 41 : e238957.

IBM SPSS Statistics 20. (2012). IBM. Software.

Lacoponi E. \& Mari, J. J. (1989). Reliability and factor structure of the Portuguese version of Self-Reporting Questionnaire. Int J Soc Psychiatry. Autumn; 35(3):213-22.

Lakatos, E. V., \& Marconi, M. A. (2010). Metodologia científica. (7a ed.), Atlas.

Leão, A. M., Gomes, I. P., Ferreira, M. J. M. \& Cavalcanti, L. P. G. (2018). Prevalência e Fatores associados à Depressão e Ansiedade entre Estudantes Universitários da Área da Saúde de um Grande Centro Urbano do Nordeste do Brasil. Revista Brasileira de Educação Médica, 42(4): 55-65.

Leão, T. M., Ianni, A. M. Z. \& Goto, C. S. (2019). Individualização e Sofrimento Psíquico na Universidade: entre a clínica e a empresa de si. Revista Humanidades \& Inovações: Universidade, pressões e adoecimento 2, 6(9): 131-143.

Lima, J. K. A. \& Brito, A. P. A. D. (2018). Desgaste e sofrimento psíquico em estudantes de medicina: uma revisão sistemática. XVII SEPA - Seminário Estudantil de Produção Acadêmica. UNIFACS, Universidade Salvador, 17: 13 p.

Mascarenhas, S. A. N., Garcia, F. M., León, G. F., Jiménez, A. C., Maciel, A. \& Roazzi, A. (2020). Habilidades conformadoras do desenvolvimento pessoal de estudantes do Ensino Superior. Revista de Psicopedagogia, Psicologia escolar e Educação. 25(2): 509-522.

Maslach, C., Schaufeli, W. B. \& Leiter, M. P. (2001). Job Burnout. Annual Review of Psychology.

Miranda, C. A., Tarasconi, C. V. \& Scortegagna, S. A. (2008). Estudo epidêmico dos transtornos mentais. Avaliação Psicológica, $7(2)$ : $249-257$.

Mucavêa, P. L. A., Abacar, M. \& Aliante, G. (2020). Burnout em estudantes de Psicologia em uma universidade pública em Moçambique. Revista Trabalho EnCena, Palmas-TO, Brasil, 5(1): 163-180.

Murakami, K. (2020). Estratégias de enfrentamento das dificuldades (“coping”) utilizadas por estudantes do Ensino Superior na área da saúde. Faculdade de Medicina de Ribeirão Preto. Ribeirão Preto.

Murakami, K., Panúncio-Pinto, M. P., Santos, J. L. F. \& Troncon, L. E. A. (2019). Estresse psicológico em estudantes de cursos de graduação da área da saúde: subsídios para promoção de saúde mental. Revista De Medicina, 98(2): 108-113.

Mussa, A., Mucavêa, P. L. A. \& Aliante, G. (2020). Burnout em estudantes de Psicologia em uma Universidade pública em Moçambique. Revista Trabalho EnCena, Tocantis, 5(1): 163-180.

Nogueira, M. J. C. (2017). Saúde mental em estudantes do Ensino Superior: fatores protetores e fatores de vulnerabilidade. Tese (Doutorado em Enfermagem) - Universidade de Lisboa, Lisboa, $269 \mathrm{f}$.

Oliveira, N. R. \& Barroso, S. M. (2020). Solidão, Depressão e Suporte Social em estudantes de Psicologia. Revista Trabalho EnCena, Tocantins, 5(1): 146162.

Padovani, R. C., Neufeld, C. B., Maltoni, J. B., Leopoldo, N. F., Souza, W. F., Cavalcanti, H. A. F., \& Lameu, J. N. (2014). Vulnerabilidade e bem-estar psicológicos do estudante universitário. Revista Brasileira de Terapias Cognitivas, 10(1): 02-10.

Pinho, A. P. M., Dourado, L. C. D. C., Aurélio, R. M. \& Bastos, A. V. B. (2013). A transição do ensino médio para a universidade: um estudo qualitativo sobre os fatores que influenciam este processo e suas possíveis consequências. Revista de Psicologia, 6(1): 33-47.

Pinho, P. S. \& Araújo, T. M. (2012). Associação entre sobrecarga doméstica e transtornos mentais comuns em mulheres. Revista Brasileira de Epidemiologia. 15(3): 560-572.

Porto, A. M. S. \& Soares, A. B. (2017). Expectativas e adaptações acadêmicas em estudantes universitários. Psicologia: Teoria e Prática. 19(1): 208-219.

Rocha, E. S. \& Sassi, A. P. (2013). Transtornos Mentais Menores entre Estudantes de Medicina. Revista Brasileira de Educação Médica, 37(2): 210-216.

Rocha, S. V., Almeida, M. M. G., Araújo, T. M. \& Júnior, J. S. V. (2010). Prevalência de transtornos mentais comuns entre residentes em áreas urbanas de Feira de Santana, Bahia. Revista Brasileira de Epidemiologia, 13(4): 630-640.

Sahão, F. T. (2019). Saúde Mental do Estudante Universitário: comportamentos que favorecem a adaptação ao Ensino Superior. Universidade Estadual de Londrina: Programa de Pós-Graduação em Análise do Comportamento.

Santos, A. F. \& Alves Junior, A. (2007). Estresse e estratégias de enfrentamento em mestrandos de ciências da saúde. Psicologia: Reflexão e Crítica, Porto Alegre, 20(1): 104-113.

Santos, A. A. A., Polydoro, S. A. J., Scortegagna, S. A. \& Linden, M. S. S. (2013). Integração ao Ensino Superior e satisfação acadêmica em universitários. Psicologia: Ciência e Profissão, 33(4): 780-793. 
Research, Society and Development, v. 10, n. 10, e483101019172, 2021

(CC BY 4.0) | ISSN 2525-3409 | DOI: http://dx.doi.org/10.33448/rsd-v10i10.19172

Santos, F. M. (2012). Análise de Conteúdo: a visão de Laurence Bardin. Revista Eletrônica de Educação, 6(1). Resenhas. Programa de Pós-Graduação em Educação.

Santos, M. A. A., Carvalho, I. F. \& Vargas, M. M. (2020). Avaliação Integrada De Escores De Qualidade De Vida E Estresse Em Pré-Vestibulandos. Aracaju: Interfaces Científicas, 8(2): 347-359.

Silus, A., Fonseca, A. L. C. \& Jesus, D. L. N. (2020). Desafios do Ensino Superior brasileiro em tempos de pandemia da Covid-19: repensando a prática docente. Liinc em Revista, 16(2): e5336.

Silva, R. S. \& Costa, L. A. (2012). Prevalência de transtornos mentais comuns entre estudantes universitários da área da saúde. Revista de Psicologia, 15(23). Souza, M., Caldas, T. \& De Antoni, C. (2017). Fatores De Adoecimento Dos Estudantes Da Área Da Saúde: uma revisão sistemática. Psicologia e Saúde em Debate, 3(1): 99-126.

Teixeira, L. A. C., Costa, R. A., Mattos, R. M. P. R. \& Pimentel, D. (2021). Saúde mental dos estudantes de Medicina do Brasil durante a pandemia da coronavirus disease 2019. Jornal Brasileiro de Psiquiatria, 70(1): 21-29.

Wang, C., Pan, R., Wan, X., Tan, Y., Xu, L., Ho, C. S. \& Ho, R. C. (2020). Respostas psicológicas imediatas e fatores associados durante o estágio inicial da epidemia de doença do coronavírus de 2019 (COVID-19) entre a população geral na China. Int. J. Environ. Res. Public Health.

World health organization. (2017). Depression and Other Common Mental Disorders. 18 p.

Xavier, A., Nunes, A. I. B. L. \& Santos, M. S. (2008). Subjetividade e sofrimento psíquico na formação do Sujeito na Universidade. Revista Mal-Estar e Subjetividade. Fortaleza, 8(2): 427-451. 